\title{
Effects of Hypoxia-Ischemia on Monoamine Metabolism in the Immature Brain
}

\author{
Faye Silverstein, MDCM, ${ }^{*}$ and Michael V. Johnston, $\mathrm{MD}^{* \dagger}$
}

\begin{abstract}
We measured acute changes in monoamine metabolites in corpus striatum of immature rat pups exposed to hypoxiaischemia, hypoxia alone, or total global ischemia. Carotid ligations and two hours of $8 \%$ oxygen environment in 7 -dayold pups led to asymmetrical turning behavior, a $70 \%$ decrease in endogenous striatal dopamine levels, and a $125 \%$ increase in homovanillic acid (HVA) concentrations on the side of ligation. In contrast, hypoxia alone and total global ischemia alone were not associated with HVA level elevation. Elevation of HVA level with hypoxia-ischemia showed a threshold effect between 1 and 1.5 hours, and this time course paralleled that for production of gross morphological changes in rats raised to maturity. The data suggest that dopamine release from striatal nerve terminals is associated with events causing brain injury during perinatal hypoxia-ischemia. Tissue HVA in the animal model appears to be a quantitative marker for the effects of the insult on a population of nerve terminals.
\end{abstract}

Silverstein F, Johnston MV: Effects of hypoxia-ischemia on monoamine metabolism in the immature brain. Ann Neurol 15:342-347, 1984

Perinatal hypoxia-ischemia triggers a cascade of biochemical events that results in neuronal injury, but the mechanisms underlying these processes are poorly understood. Various combinations of hypoxia and ischemia probably lead to final common reactions that disrupt intraneuronal metabolic machinery and produce cell death $[16,22]$. To study the acute responses of immature neurons to this form of injury, we measured changes in monoamine metabolism in the corpus striatum of rats exposed to hypoxia-ischemia, hypoxia alone, or total global ischemia. A dense dopamine innervation appears early in this region $[4,5,10,23]$, and its synaptic neurochemical markers might be expected to reflect neuronal injury. The rat pup model of unilateral hypoxia-ischemia used in the experiments has been shown to result in a reproducible pattern of neuronal damage $[9,17]$. Furthermore, its severity can be manipulated by varying the length of the hypoxic period. The results of the present experiments demonstrate that severe perinatal hypoxia-ischemia has an effect on dopamine terminals that differs markedly from the effect of hypoxia or total ischemia alone.

\section{Methods \\ Pregnant Sprague-Dawley rats (Charles River) were housed in separate cages after 17 days' gestation, and pups were born on day 21 or 22 of gestation. One hundred eighty-five 7 -day- old pups from twenty-one litters were used for the ex- periments.}

To produce unilateral hypoxia-ischemia, 137 pups were briefly anesthetized with ether and a carotid artery was ligated. In 48 sham-operated control pups, the artery was manipulated but not ligated. After two hours in which pups were allowed to suckle with the dam, they were placed in a glass chamber warmed to $37^{\circ} \mathrm{C}$ and supplied with humidified $8 \%$ oxygen (balance, nitrogen). Pups were removed to room air $0.5,1,1.5$, or 2 hours later, and killed after 5 to 10 minutes. Results were unaffected by a delay in death of up to 10 minutes. Untreated pups and animals exposed to $8 \%$ oxygen alone for 2 hours were also studied.

Upon removal of the animals from the chamber, the presence or absence of spontaneous turning behavior was noted. If strong unilateral turning was not present, the response to tail pinch was noted. Pups were killed by decapitation, and the corpora striata were dissected from the brain at $4^{\circ} \mathrm{C}$, frozen on dry ice, weighed, and stored in a $-70^{\circ} \mathrm{C}$ freezer. To measure the animals' physiological response, groups of animals were removed at intervals during hypoxia, and blood from severed neck vessels was analyzed for partial pressure of oxygen and carbon dioxide, and pH (Radiometer, Copenhagen). In some animals arterial samples were collected from a severed carotid artery or the left ventricle. Blood gas findings were similar in carotid-ligated and sham-operated rats.

To examine the effects of total global ischemia on monoamine metabolism, pups were decapitated and the heads were placed in beakers at $37^{\circ} \mathrm{C}$ for periods of time similar to those used for hypoxia in the ligated pups. Brains were then dissected and analyzed identically to the hypoxic-ischemic ones.

Striatal concentrations of dihydroxyphenylacetic acid
From the *Departments of Pediatrics and Neurology, the University of Michigan Medical Center, and the ${ }^{t}$ Center for Human Growth and Development, the University of Michigan, Ann Arbor, MI 48109 .
Received May 19, 1983, and in revised form Aug 23. Accepted for publication Aug 29, 1983.

Address reprint requests to Dr Johnston, the University of Michigan, Neuroscience Laboratory Bldg, 1103 E Huron St, Ann Arbor, MI 48109. 
(DOPAC) and homovanillic acid (HVA), metabolites of dopamine, and 5-hydroxyindoleacetic acid (5-HIAA), the major acid metabolite of serotonin, were measured using high-performance liquid chromatography with electrochemical detection (HPLC-EC) [1]. The HPLC system included a C-18 column (Whatman) and an electrochemical detector (Bioanalytical Systems glassy carbon electrode, potential maintained at $+0.78 \mathrm{~V}$, run at sensitivities of 1 to $2 \mathrm{nA}$ ). The mobile phase was a methanol and water mixture containing $0.01 \mathrm{M}$ sodium acetate and $0.0001 \mathrm{M}$ ethylenediaminetetraacetate, and $\mathrm{pH}$ adjusted to 3.7. Tissue was homogenized by sonication in $250 \mu \mathrm{l}$ of $0.1 \mathrm{M}$ perchloric acid containing $0.1 \%$ sodium metabisulfite to impede oxidation of the metabolites. Seventy-five $\mu$ l of $1 \mathrm{M}$ Tris, pH 8.6, was added to the suspension to enhance precipitation of particulate matter [27]. After centrifugation of the suspension at 18,000 rpm for 15 minutes, 40 to $80 \mu \mathrm{l}$ aliquots of supernatant were analyzed. Concentrations of DOPAC, HVA, and 5-HIAA were calculated using external standards, and values were expressed per milligram of tissue. Endogenous dopamine was measured in perchloric acid extracts with HPLC-EC using a cation exchange column.

For correlation of the time course of short-term changes in monoamine metabolism with later morphological effects, a group of animals was raised to 7 weeks of age and then killed. The forebrain was separated from the pons-medulla and cerebellum, and the left and right cerebral hemispheres were weighed separately.

\section{Results}

Eighty-three percent of pups survived carotid artery ligation and up to 2 hours of hypoxia, whereas $98 \%$ of

Fig 1. Rat pups demonstrating turning behavior after carotid ligation and 2 bours of bypoxia. The 8 pups on the right bad right carotid ligation. whereas the 6 on the left had left carotid ligation. the sham-operated animals survived hypoxia. Immediately after emerging from the hypoxic chamber and for about 10 minutes thereafter, $66 \%$ of the ligated pups turned either spontaneously or after tail pinching toward the side of ligation (Fig 1), whereas $6 \%$ turned to the opposite side. Of the remaining pups $(28 \%)$, half were lethargic and did not turn and the rest showed no turning preference.

Blood gas findings remained stable and uniform over the period of hypoxia. The partial pressure of oxygen fell abruptly to approximately 30 torr, whereas that of carbon dioxide fell to 25 torr because of hyperventilation. The pups also experienced a mild, gradual fall in arterial $\mathrm{pH}$ during the period in the hypoxic atmosphere.

\section{Neurochemical Changes}

The pups' ipsilateral turning behavior after the hypoxic-ischemic insult suggested an asymmetry in striatal dopamine concentration between the two sides [24]. Assay of endogenous dopamine verified this speculation; the concentration of this neurotransmitter was reduced by $70 \%$ on the ligated side after 2 hours of hypoxia (Fig 2). No difference was found berween the unligated side at 2 hours and striata in untreated controls.

Because the reduced dopamine concentration might have been related to decreased synthesis or increased nerve terminal release and degradation, the dopamine metabolites DOPAC and HVA were assayed. The metabolite for serotonin, 5-HIAA, was also assayed to assess another population of monoaminergic nerve terminals (Table).

In pups with unilateral carotid ligation exposed to 0.5 or 1 hour of the $8 \%$ oxygen atmosphere, there was

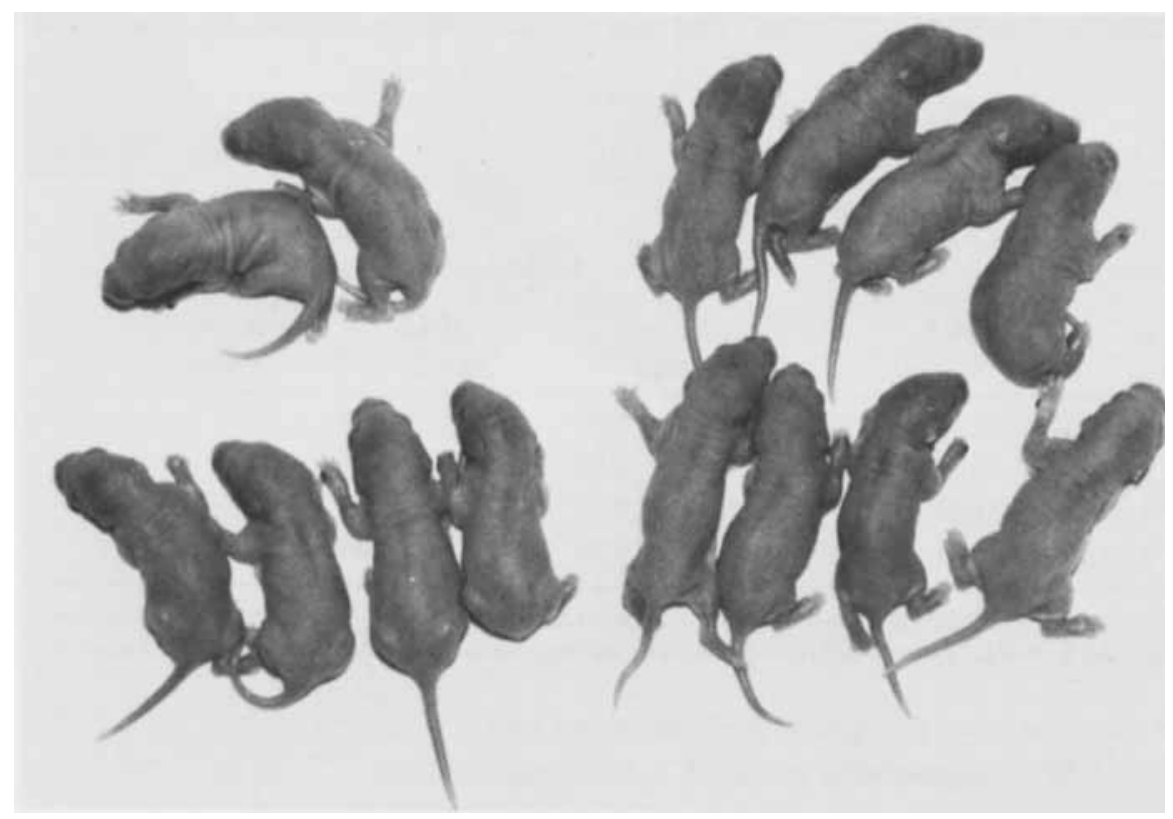




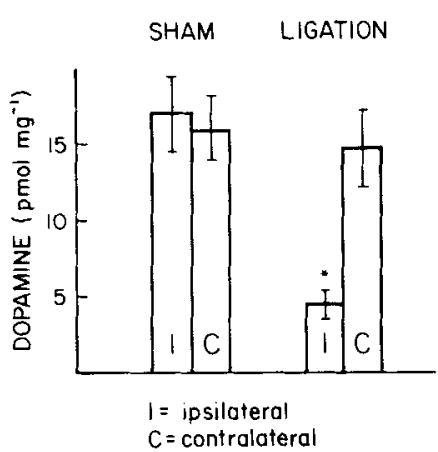

Fig 2. Comparison of striatal dopamine concentration bilaterally in bypoxic-ischemic pups and sham-operated bypoxic controls. There were 12 animals in each group. Ipsilateral and contralateral refer to side of surgery. Bar beight indicates mean \pm SEM. Asterisk indicates $\mathrm{p}<0.01$ using Student $\mathrm{t}$ test for paired values.

little change in monoamine metabolites on either side of the brain. After 1.5 hours of hypoxia, however, ipsilateral HVA concentrations rose to $60 \%$ above baseline values and were $70 \%$ higher than those in the contralateral striatum. At 2 hours the ipsilateral concentration had risen to $125 \%$ above control values and was $64 \%$ above the level on the other side. At 2 hours of hypoxia-ischemia, HVA levels on the side opposite that of ligation were $37 \%$ above baseline values $(p<$ 0.001 ). In contrast to HVA, there was no significant change in the concentration of the other dopamine metabolite, DOPAC, or the serotonin metabolite, 5 HIAA.

To determine how much of the rise in HVA level was related to effects of hypoxia rather than effects of hypoxia plus ischemia, a group of pups was studied during hypoxia without carotid ligation (Fig 3A). Hypoxia alone produced a $30 \%$ fall in HVA and a $46 \%$ fall in 5-HIAA level by 2 hours. DOPAC concentrations were unaffected. Total global ischemia produced

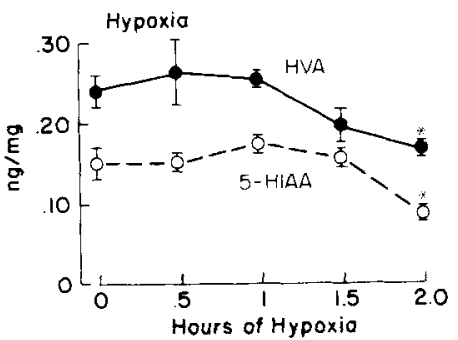

A

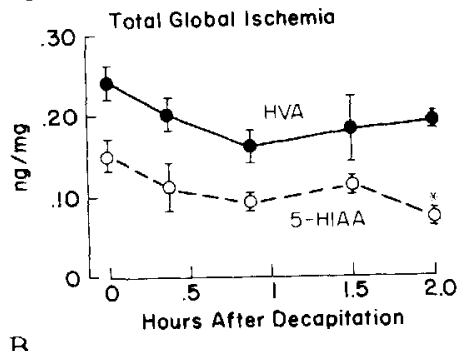

B

Fig 3. (A) Time course of changes in striatal concentrations of bomovanillic acid (HVA) and 5-bydroxyindoleacetic acid (5HIAA) with increasing duration of hypoxia in 7-day-old pups. There were 4 to 14 animals in each group, except at 0.5 hour $(2$ animals). (B) Time course of changes in striatal concentrations of HVA and 5-HIAA with increasing duration of total global ischemia. There were 3 to 4 animals in each group. All values are mean \pm SEM. Asterisk indicates $\mathrm{p}<0.01$ using Student $\mathrm{t}$ test for paired values.

similar results, although HVA level did not fall by 2 hours, as it did during hypoxia (Fig 3B). With total global ischemia, values for HVA and DOPAC were not significantly different at 2 hours, whereas 5-HIAA values fell by $53 \%$ in the same period.

That the $8 \%$ oxygen environment alone reduced HVA concentrations in the immature striatum suggests that the higher values obtained from the ligated, hypoxic pups reflect the contribution of relative ischemia. The actual impact of the carotid ligation in the

Striatal Concentrations of Metabolites in Ligated Pups

Side of Measurement

\begin{tabular}{|c|c|c|c|c|c|c|}
\hline \multirow[b]{3}{*}{$\begin{array}{l}\text { Hours of } \\
\text { Hypoxia }\end{array}$} & \multicolumn{6}{|c|}{ Side of Measurement } \\
\hline & \multicolumn{3}{|c|}{ Ipsilateral to Ligation } & \multicolumn{3}{|c|}{ Contralateral to Ligation } \\
\hline & $\begin{array}{l}\text { 5-HIAA } \\
(\mathrm{ng} / \mathrm{mg})\end{array}$ & $\begin{array}{l}\text { DOPAC } \\
(\mathrm{ng} / \mathrm{mg})\end{array}$ & $\begin{array}{l}\text { HVA } \\
(\mathrm{ng} / \mathrm{mg})\end{array}$ & $\begin{array}{l}\text { 5-HlAA } \\
(\mathrm{ng} / \mathrm{mg})\end{array}$ & $\begin{array}{l}\text { DOPAC } \\
(\mathrm{ng} / \mathrm{mg})\end{array}$ & $\begin{array}{l}\text { HVA } \\
(\mathrm{ng} / \mathrm{mg})\end{array}$ \\
\hline $0.5(n=6)$ & $0.17 \pm 0.02$ & $0.10 \pm 0.03$ & $0.21 \pm 0.05$ & $0.15 \pm 0.02$ & $0.12 \pm 0.03$ & $0.21 \pm 0.03$ \\
\hline $1.0(\mathrm{n}=16)$ & $0.17 \pm 0.02$ & $0.11 \pm 0.07$ & $0.20 \pm 0.04$ & $0.15 \pm 0.02$ & $0.07 \pm 0.06$ & $0.15 \pm 0.02$ \\
\hline $1.5(\mathrm{n}=17)$ & $0.16 \pm 0.02$ & $0.13 \pm 0.02$ & $0.39 \pm 0.05^{b}$ & $0.16 \pm 0.01$ & $0.16 \pm 0.03$ & $0.23 \pm 0.02$ \\
\hline $2.0(\mathrm{n}=31)$ & $0.12 \pm 0.01$ & $0.14 \pm 0.01$ & $0.54 \pm 0.04^{c}$ & $0.11 \pm 0.01$ & $0.22 \pm 0.04$ & $0.33 \pm 0.03$ \\
\hline
\end{tabular}

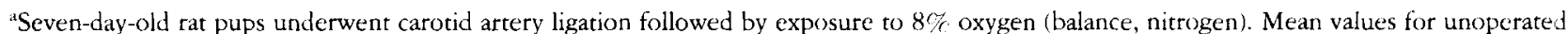
controls were the same on both sides: DOPAC, $0.13 \pm 0.02 \mathrm{ng} / \mathrm{mg} ; \mathrm{HVA}, 0.24 \pm 0.02 \mathrm{ng} / \mathrm{mg} ; 5-\mathrm{HIAA}, 0.15 \pm 0.02 \mathrm{ng} / \mathrm{mg}$. Values are expressed as means $\pm \mathrm{SEM}$

Statistical significance: ${ }^{b} p<0.01 ; ' p<0.001$ comparing ligated and opposite side using Student $t$ test.

5-HIAA $=$ 5-hydroxyindoleacetic acid; DOPAC $=$ dihydroxyphenylacetic acid $; \mathrm{HVA}=$ homovanillic acid. 


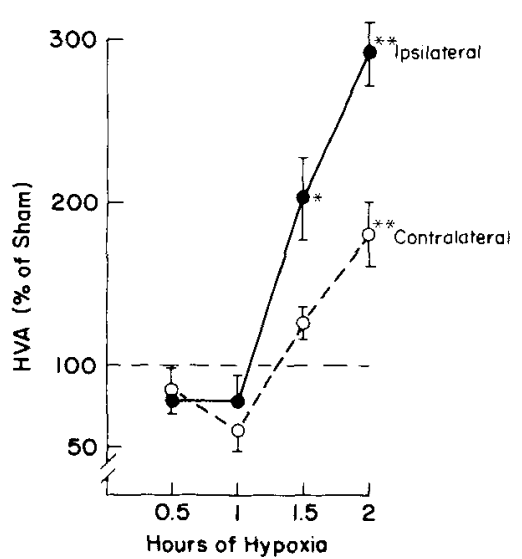

Fig 4. Time course of changes in striatal concentration of bomovanillic acid (HVA) in ligated, bypoxic pups, with values expressed as a percentage of concentrations in sham-operated pups exposed to the same duration of $8 \%$ oxygen. Values on the ligated side were significantly bigher than those on the opposite side at 1.5 and 2 hours (see Table). Ipsilateral and contralateral refer to the side of ligation. Values are means $\pm S E M$. Asterisk indicates $\mathrm{p}<0.01$, and double asterisk indicates $\mathrm{p}<0.001$; comparisons were made using Student t test ( $n=6$ to 31 ).

face of hypoxia is shown in Figure 4, where values for the hypoxic-ischemic pups are plotted in relationship to those for pups exposed to sham operation and hypoxia alone. Although HVA concentrations on both sides are slightly below those in hypoxic pups at 0.5 and 1 hour, there is a sharp rise on both sides at 1.5 and 2 hours. By 2 hours of hypoxia, HVA levels on the ligated side rose to $300 \%$ of values in sham-operated hypoxic pups, whereas those on the opposite side were $180 \%$ of the sham levels.

The time course for elevation of HVA levels in the hypoxic-ischemic brain suggests that there is a threshold of hypoxic-ischemic exposure between 1 and 1.5 hours when some critical events lead to increased turnover of dopamine. To determine whether this timing corresponded to a threshold for morphological changes, hemisphere mass was measured as a readily quantifiable though gross index of injury in the ligated hypoxic pups that had matured. Hemispheral atrophy is a highly reproducible lesion in pups exposed for 2 hours. Figure 5 demonstrates that the time threshold for production of this effect lies between 1 and 1.5 hours. Thus, the time course and threshold for later, grossly obvious brain injury parallels that observed for the acute increase in striatal HVA concentration.

\section{Discussion}

The perinatal brain is relatively resistant to hypoxia [8]. Clinical and experimental animal studies suggest that reduced blood flow combined with hypoxia is required to produce morphologically detectable brain injury [3]. However, little is known at a biochemical level about how hypoxia and ischemia interact to worsen damage.

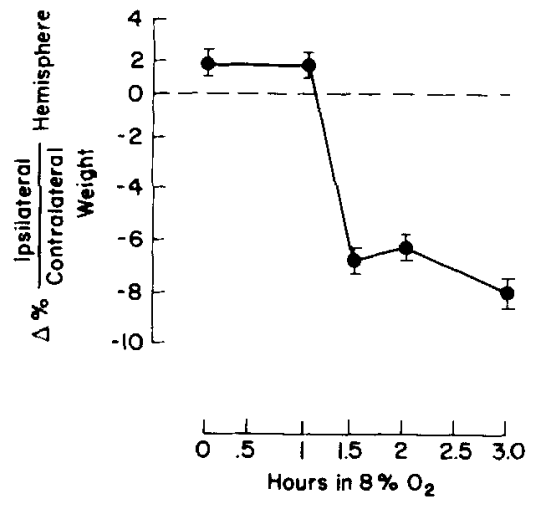

Fig 5. Relationship between reduction in ipsilateral bemisphere mass and duration of $8 \%$ oxygen exposure in 20 rat pups with unilateral carotid artery ligation. The animals were raised to 7 weeks of age before being killed, and the weights of the two bemispheres were compared. The hemispheres contralateral to the ligation were the same weight as in untreated controls after each period of exposure (weight, $640 \pm 12 \mathrm{mg}$ ).

Other experiments have examined changes in metabolic substrates, organic acids, and acid-base balance produced by combinations of hypoxia and ischemia $[22,25,29]$. Our approach in the present experiments has focused on quantitating the response of a group of immature nerve terminals to the insult.

The model of perinatal hypoxia-ischemia in 7-dayold rats is a variation of the Levine procedure [12]. Carotid ligation or hypoxia alone produces no injury, but the combination damages the ipsilateral hemisphere. In the adult rat preparation of Levine, unilateral carotid artery ligation performed during a period of severe hypoxia has been shown to limit reactive hyperemia in the ipsilateral hemisphere [20]. Although blood flow increases four- to sixfold in response to hypoxia on the unligated side, it rises by only half as much on the ligated side. Thus the hemisphere damage is related to relative ischemia on the ligated side in the face of hypoxia. Carotid ligation plus hypoxia may act through a similar mechanism in the infant rats. Blood flow in the preparation has not yet been accurately measured, however, because of the small size of rat pups.

The preparation produced predominantly unilateral brain injury that has been histologically studied immediately after insult [17] and after the animals have matured to 3 and 7 weeks of age [9]. The brainstem and cerebellum are relatively spared. Unlike the adult rat experiments using the Levine procedure, in which animals usually die after a short period, in these studies the immature rats may be studied at intervals of hypoxia spanning several hours. As in the adults, the cerebral cortex, hippocampus, and striatum are sensi- 
tive to injury. However, the periventricular zone in the corpus striatum is damaged more than the rest of the hemisphere at 2 hours. Longer periods are required to cause cavitations in the overlying cortex [9].

By varying the duration of $8 \%$ oxygen exposure, we found a threshold for major morphological injury that lies between 1 and 1.5 hours. Although the freely breathing animals were not physiologically controlled, their arterial blood gas values indicate that there was no major cardiorespiratory instability throughout the period of the experiment. Welsh and colleagues [29] obtained similar results for nonligated hypoxic pups treated similarly to ours. They also found only modest decreases in pulse rate and blood pressure over 2 hours. Therefore, the threshold for brain injury in the preparation is probably related to metabolic factors within the brain.

Previous work showed that dopamine levels are reduced in brains of monkeys and gerbils on the side of complete strokes [31]. We studied dopamine metabolism in striatum in the hypoxic-ischemic pups because of the turning behavior and also because this region is a major site of injury in the model. The duration of hypoxia with carotid ligation that produced severe tissue injury corresponded to the time when the surge in striatal HVA occurred. This observation suggests that factors responsible for the tissue injury and those producing the rise in HVA concentration are linked in some way.

The elevation of HVA level could result from increased synthesis and turnover of dopamine, exaggerated release and degradation, or decreased clearance from the striatum. Diminished clearance from tissue seems unlikely, because 5-HIAA, which is cleared by similar mechanisms, was unaffected. Augmented synthesis would not be expected, because tyrosine hydroxylase, the rate-limiting synthetic enzyme for dopamine, has a cofactor requirement for molecular oxygen. Increased synthesis probably does not play a role, because dopamine was in fact reduced by $70 \%$ after 2 hours. Furthermore, study of the fate of tritiated catecholamines injected into gerbils that had suffered strokes suggests that dopamine synthesis is reduced and release is augmented [11]. The most plausible interpretation of our results is that elevated HVA levels are produced by a massive release of dopamine from nerve terminals in response to severe hypoxia-ischemia.

Although the rise in HVA level was markedly greater on the ligated side, a significant elevation occurred on the side opposite carotid ligation as well. This finding may represent some crossover of the hypoxic-ischemic effect. Approximately $10 \%$ of pups in a large series have been noted to have bilateral morphological changes (M. V. Johnston, unpublished data, 1982). Alternately, the contralateral changes may reflect crosstalk from the opposite nigrostriatal circuit rather than a primary striatal effect of the insult [15]. Although cortical catecholamine neurons in the rat may respond differently to stroke depending on whether the right or left hemisphere is involved [18], the side of the ligation made no difference in the HVA response in our experiment.

Although HVA levels rose during hypoxia-ischemia, DOPAC and 5-HIAA concentrations remained unchanged. There are several possible reasons for the DOPAC results. This metabolite is formed primarily by monoamine oxidase from free dopamine within presynaptic nerve terminals. In contrast, HVA is a product of dopamine released to the outside of the nerve terminal, where catechol-o-methyltransferase and monoamine oxidase are both located. DOPAC may not rise because of reduced activity of the oxygen-dependent enzyme monoamine oxidase. Because monoamine oxidase is required for production of both metabolites, however, the more likely explanation is that hypoxiaischemia stimulates extraneuronal release of dopamine predominantly. The lack of effect on 5-HIAA levels suggests, as has previous work, that serotonin nerve endings are less sensitive to ischemia than are catecholamine terminals $[11,13]$.

Hypoxia-ischemia severe enough to cause tissue injury affected dopamine metabolism quite differently from hypoxia or global ischemia alone. However, up to 1 hour of hypoxia plus ligation produced a small reduction in HVA resembling the effect of hypoxia alone. This finding, along with previous work, suggests that mild hypoxia or total ischemia causes a reduction in catecholamine turnover $[7,31]$. Longer periods of hypoxia-ischemia probably induce massive release of nerve terminal dopamine. Whether this release is associated with accelerated neuronal impulse flow is unclear. In the gerbil stroke model, the fall in dopamine levels is associated with the occurrence of seizures. Although the rat pups were tremulous, they did not appear overtly to have seizures [28]. Nevertheless, it is possible that one of the critical events occurring at the time of injury is localized neuronal depolarization. Convulsions in human neonates with hypoxia-ischemia correlate strongly with brain injury [19, 21, 26]. Although it may seem paradoxical that hypoxia-ischemia produces an effect of injury that total ischemia does not, morphological studies indicate that the brain may withstand total cessation of flow better than partial ischemia $\{14,22\}$. Also, in infant monkeys, prolonged partial asphyxia may produce more damage than total asphyxia with resuscitation [14].

The data from these experiments suggest that dopamine release is associated with events causing neuronal injury. Furthermore, the HVA level in tissue may be a quantitative index of the severity of the insult. Because neurotransmitter release is calcium dependent 
[6], one reasonable possibility is that HVA may reflect hypoxia-ischemia-induced calcium entry into nerve terminals. Elevated intracellular calcium levels from a massive influx are thought to mediate hypoxic cell death in several systems [16, 22]. Another possible link between the elevated dopamine release and injury is that the dopamine is in itself noxious to surrounding neurons [30]. Release of another neurotransmitter, glutamate, has been suggested to play a role in hypoxicischemic injury [2]. Our studies suggest that HVA assay in this model can serve as a rapid quantitative indicator of effects of metabolic variables (e.g., blood gases, glucose), as well as other permutations of hypoxia and ischemia and drugs.

Supported by Grant NS RO1 019678 from the Narional Institutes of Health, the United Cerebral Palsy Research and Education Foundation, and Teacher-Investigator Award K07NS00603 (to M. V. J.).

Dr Silverstein is the recipient of a postdoctoral fellowship from the Medical Research Council of Canada.

\section{References}

1. Anderson GM, Young JB, Cohen DJ: Rapid chromatographic determination of tryptophan, tyrosine, 5-hydroxyindoleacetic acid and homovanillic acid in cerebrospinal fluid. J Chromatogr 164:501-505, 1979

2. Bosley TM, Woodhams PL, Gordon RD, Balazs R: Effect of anoxia on the stimulated release of amino acid neurotransmitters in the cerebellum in vitro. J Neurochem 40:189-201, 1983

3. Brierley JB, Meldrum BS, Brown AW: The threshold of cerebral anoxic-ischemic cell change. Arch Neurol 29:367-373, 1973

4. Cheronis JC, Erinoff L, Heller A, Hoffman PC: Pharmacological analysis of the functional ontogeny of the nigrostriatal dopaminergic neurons. Brain Res 169:545-560, 1979

5. Coyle JT, Campochiaro P: Ontogenesis of dopamine cholinergic interactions in the rat striatum: a neurochemical study. J Neurochem 27:673-678, 1976

6. Drapeau P, Blaustein MP: Initial release of ${ }^{3} \mathrm{H}$-dopamine from rat striatal synaptosomes: correlation with calcium entry. J Neurosci 3:703-713, 1983

7. Hedner T: Central monoamine metabolism and neonatal oxygen deprivation. Acta Physiol Scand [Suppl] 460:1-34, 1978

8. Jilek $\mathrm{L}$ : The reaction and adaptation of the central nervous system to stagnant hypoxia and anoxia during ontogeny. In Himwich WA (ed): Developmental Neurology. Springfield, IL, Thomas, 1970, pp 331-369

9. Johnston MV: Neurotransmitter alterations in a model of perinatal hypoxic-ischemic brain injury. Ann Neurol 13:511518,1983

10. Johnston MV, Coyle JT: Development of central neurotransmitter systems. In Elliott $\mathrm{K}$, Wilson J (eds): The Fetus and Independent Life. Ciba Symposium 86, London, Pitman, 1981, pp 251270
11. Lavyne $\mathrm{MH}$, Moskowitz $\mathrm{MA}$, Larin $\mathrm{F}$, et al: Brain $\mathrm{H}^{3}$ catecholamine metabolism in experimental cerebral ischemia. Neurology (Minneap) 25:483-485, 1975

12. Levine S: Anoxic-ischemic encephalopathy in rats. Am J Pathol $36: 1-17,1960$

13. Lust WD, Mrsulja BB, Branislava JM, et al: Putative neurotransmitters and cyclic nucleotides in prolonged ischemia of the cerebral cortex. Brain Res 98:394-399, 1975

14. Myers RE: Experimental models of perinatal brain damage: relevance to human pathology. In Gluck L (ed): Intrauterine Asphyxia and the Developing Fetal Brain. Chicago, Year Book, 1977, pp 37-97

15. Nieoullon A, Cheramy A, Glowinski J: Interdependence of the nigrostriatal dopaminergic systems on the two sides of the brain in the cat. Science 198:416-418, 1977

16. Raichie ME: The pathophysiology of brain ischemia. Ann Neurol 13:2-10, 1983

17. Rice JE III, Vannucci RC, Brierley JB: The influence of immaturity on hypoxic-ischemic brain damage in the rat. Ann Neurol 9:131-141, 1981

18. Robinson RG, Shoemaker WJ, Schlumpf M: Time course changes in catecholamines following right hemisphere cerebral infarction in the rat. Brain Res 181:202-208, 1980

19. Rose AL, Lombroso CT: Neonatal seizure states: a study of clinical, pathological and electroencephalographic features in 137 full term babies with a long term follow-up. Pediatrics 45:404-425, 1970

20. Salford LG, Siesjo BK: The infuence of arterial hypoxia and unilateral carotid artery occlusion upon regional blood flow and metabolism in the rat brain. Acta Physiol Scand 92:130-141, 1974

21. Scott H: Outcome of very severe birth asphyxia. Arch Dis Child 51:712-716, 1976

22. Siesjo BK: Cell damage in the brain: a speculative synthesis. J Cerebral Blood Flow Metab 1:155-185, 1981

23. Tennyson VM, Barrett RE, Cohen G, et al: The developing neostriatum of the rabbit: correlation of fluorescence, histochemistry, electron microscopy, endogenous dopamine levels and $\left({ }^{3} \mathrm{H}\right)$ dopamine uptake. Brain Res 46:251-285, 1972

24. Ungerstedt $U$ : Functional dynamics of central monoamine pathways. In Schmitt FO, Worden FG (eds): The Neuroscience Third Study Program. Boston, MIT Press, 1974, pp 979-988

25. Vannucci RC, Vannucci SJ: Cerebral carbohydrate metabolism during hypoglycemia and anoxia in newborn rats. Ann Neurol 4:73-79, 1978

26. Volpe JJ: Neurology of the Newborn. Philadelphia, Saunders, 1981, pp 125-127

27. Walsh FX, Stevens TJ, Langlais PJ, Bird ED: Dopamine and homovanillic acid concentrations in striatal and limbic regions of human brain. Ann Neurol 12:52-55, 1982

28. Welch KMA, Wang T-PF, Chabi E: Ischemia-induced seizures and cortical monoamine levels. Ann Neurol 3:152-155, 1978

29. Welsh FA, Vannucci RC, Brierley JB: Columnar alterations of NADH fluorescence during hypoxia-ischemia in immarure rat brain. J Cerebral Blood Flow Metab 2:221-228, 1982

30. Wurtman RJ, Zervas NT: Monoamine neurotransmitters and the pathophysiology of stroke and central nervous system trauma. J Neurosurg 40:34-36, 1974

31. Zervas NT, Hari $H$, Negora $M$, et al: Reduction in brain dopamine following experimental cerebral ischemia. Nature 247:283-284, 1974 